\title{
Vascular Endothelial Growth Factor-A in Lactobacillus Casei Cell Wall Extract-Induced Coronary Arteritis of a Murine Model
}

\author{
I-Chun Lin, MD, PhD; Jiunn-Ming Sheen, MD; You-Lin Tain, MD, PhD; \\ Ming-Huei Chou, PhD; Li-Tung Huang, MD; Kuender D. Yang, MD, PhD
}

\begin{abstract}
Background: Vascular endothelial growth factor (VEGF) is associated with Kawasaki disease (KD), the most commonly acquired heart disease in developed countries. This study investigated the involvement of VEGF-A expression and its related signaling pathway in Lactobacillus casei cell wall extract (LCWE)-induced murine coronary artery lesions (CALs), and analyzed this in regard to the inhibition of CALs by spleen tyrosine kinase (Syk).
\end{abstract}

Methods and Results: Wild-type BALB/C mice were intraperitoneally injected with LCWE (1 mg/ml) to induce CALs. The aortic roots, ventricular myocardium, peripheral blood leukocytes (PBLs), spleen, liver, kidneys, and lungs were analyzed for VEGF-A expression. Phosphate buffered saline (PBS)-, lipopolysaccharide (LPS)-, and zymosan-treated mice served as controls, and an oral Syk inhibitor served as an arteritis-ameliorated reagent. In aortic roots and PBLs, LCWE induced an early upregulation and a late downregulation of VEGF-A expression. No differential VEGFA expression was observed in the other organs. Most importantly, Syk inhibition significantly attenuated the LCWEinduced expression of VEGF-A, dimethylarginine dimethylaminohydrolase (DDAH)-1, and endothelial nitric oxide synthase in aortic roots. However, LCWE-induced aortic DDAH-2 expression remained higher, despite Syk inhibition.

Conclusions: Local VEGF-A and its signaling pathway are associated with the development of LCWE-induced CALs. Therefore, the clinical correlation between VEGF and human KD and the role of the VEGF-A regulation and signaling pathway in murine CALs warrant further investigation. (Circ $J$ 2014; 78: 752-762)

Key Words: Coronary artery lesion; Kawasaki disease; Spleen tyrosine kinase; Vascular endothelial growth factor

$\mathbf{K}$ awasaki disease $(\mathrm{KD})$ is the most common cause of systemic vasculitis in children and affects mediumand large-sized blood vessels throughout the body. With increasing prevalence, coronary artery lesions (CALs) associated with KD contribute to the leading cause of acquired heart disease in childhood in developed countries. KD is characterized by an initial robust immune response followed by the development of inflammatory CALs of unknown etiology., ${ }^{1,2}$ A large amount of clinical and pathological evidence has indicated that persistently chronic vascular remodeling occurs in such CALs. ${ }^{3-7}$ However, the underlying mechanism by which systemic immune activation progresses to leukocyte infiltration and continuing vascular remodeling of CALs remains unknown.

Vascular endothelial growth factor (VEGF), which has dif- ferent subtypes, plays an essential role in vascular remodeling and angiogenesis during vascular development and disease.,89 VEGF-A, previously known as VEGF, is expressed in various cell types, including lymphocytes, neutrophils and macrophages, as well as smooth muscle cells and cardiomyocytes. It is capable of acting as a proinflammatory cytokine by increasing the permeability of endothelial cells and inducing the expression of endothelial adhesion molecules, thus acting as a chemoattractant of monocytes ${ }^{10-12}$ and macrophages. ${ }^{13}$ Several peripheral immune studies have provided evidence of a relationship between VEGF and KD. ${ }^{14-19}$ VEGF-A has been suggested to contribute to vascular leakage in $\mathrm{KD}$, leading to hypoalbuminemia and increased microvascular permeability. ${ }^{20}$ Serum levels of VEGF-A markedly increase during the acute phase of $\mathrm{KD}$, with much higher levels in patients with

Received May 15, 2013; revised manuscript received November 5, 2013; accepted November 7, 2013; released online December 14, 2013 Time for primary review: 18 days

Department of Pediatrics, Kaohsiung Chang Gung Memorial Hospital and Chang Gung University College of Medicine, Kaohsiung (I.-C.L., J.-M.S., Y.-L.T., L.-T.H.); Graduate Institute of Clinical Medical Sciences, College of Medicine, Chang Gung University, Kaohsiung (M.-H.C.); and Department of Medical Research, Show Chwan Memorial Hospital in Chang Bing, Changhua (K.D.Y.), Taiwan

Mailing address: Kuender D. Yang, MD, PhD, Department of Medical Research, Show Chwan Memorial Hospital in Chang Bing, 6-1 Lu-Kung Road, Chang Bing Industrial Park, Lu-Kang, Changhua 505, Taiwan. E-mail: yangkd.yeh@hotmail.com or Li-Tung Huang, MD, Department of Pediatrics, Kaohsiung Chang Gung Memorial Hospital, 123 Ta-Pei Road, Niao-Sung, Kaohsiung 833,

Taiwan._E-mail: huang_li@pie.com.tw

ISSN-1346-9843 doi:10.1253/circj.CJ-13-0612

All rights are reserved to the Japanese Circulation Society. For permissions, please e-mail: cj@j-circ.or.jp 
CALs than those without CALs. ${ }^{18,19}$ Ohno et al ${ }^{17}$ have suggested that serum VEGF levels, together with a duration of fever, are powerful predictors of the development of coronary aneurysms. In a genomics study, a significant association was found between 2 single nucleotide polymorphisms in Vegf and KD susceptibility, suggesting that VEGF dysregulation contributes to the disruption of vascular homeostasis in KD. ${ }^{21}$ VEGF can be regulated by oxidative stress. ${ }^{22}$ Patients with KD have been reported to have hyperoxidant stress during the acute stage, which improved after intravenous immunoglobulin treatment. ${ }^{23,24}$ Taken together, these associations of VEGF with KD suggest that VEGF-A plays a role in the pathogenesis of CALs.

Recently, we have demonstrated that spleen tyrosine kinase (Syk) is involved in Lactobacillus casei cell wall extract (LCWE)-induced murine CALs and proinflammatory productions. ${ }^{25}$ Some studies have suggested the participation of Syk in the regulation and signaling pathway of VEGF, ${ }^{26,27}$ and a potential therapeutic application of Syk for inflammatory vascular injury. ${ }^{28}$ Therefore, by using a mouse model of coronary arteritis induced by LCWE, which mimics CALs in human KD patients, ${ }^{29}$ we examined the microenvironment of VEGFA expression and alteration in the aortic roots, ventricular myocardium (VM), and in other organs and tissues, as well as the related signal pathway in response to Syk inhibition.

\section{Methods}

\section{LCWE Preparation}

LCWE was prepared as previously described. ${ }^{30}$ In brief, cells of L. casei (ATCC 11578; Bioresource Collection and Research Center, Taiwan), were cultured in Lactobacillus MRS broth (Difco; Detroit, MI, USA) at $37^{\circ} \mathrm{C}$. After being harvested, the cells were sequentially treated with $4 \%$ SDS, RNase, DNaseI, and trypsin (all from Sigma-Aldrich, St. Louis, MO, USA), and then finally sonicated at a $20-\mathrm{kHz}$ frequency (Vibra Cell $^{\mathrm{TM}}$, Sonics \& Materials Inc, Newtown, CT, USA). After centrifugation at $20,000 \mathrm{~g}$, the supernatant was quantified based on the concentration of its rhamnose content by using a phenolsulfuric acid colorimetric assay. ${ }^{31}$

\section{Mice and a Mouse Model of Coronary Arteritis}

Wild-type male BALB/c mice (National Laboratory Animal Center, Taiwan) were used in this study. All animal experiments were performed in strict accordance with legislation on the protection of animals, and the protocol was also approved (Permit number: 2009071301, 2007032602) by the Animal Care Committee in Kaohsiung Chang Gung Memorial Hospital. All procedures were performed under anesthesia, and all efforts were made to minimize suffering.

To induce a mouse model of CALs, 4- to 5-week-old $\mathrm{BALB} / \mathrm{c}$ mice were intraperitoneally injected with $1 \mathrm{ml}$ of PBS containing $1 \mathrm{mg}$ of LCWE, or with $1 \mathrm{ml}$ of phosphate buffered saline (PBS), lipopolysaccharide (LPS) $(200 \mu \mathrm{g} / \mathrm{ml}$; SigmaAldrich) or zymosan (100 mg/ml; Sigma-Aldrich) as controls. ${ }^{25}$ LPS was reported to cause no induction of CALs in mice. ${ }^{32}$ For in vivo arteritis-ameliorated experiments, BAY 61-3606 hydrochloride hydrate (BAY, a specific Syk inhibitor; SigmaAldrich) was administrated as previously prescribed. ${ }^{25}$ In the BAY-treated (BAY+LCWE) group, the severity of the arteritis was significantly reduced. ${ }^{25}$ Several organs, including the aortic roots and ventricular myocardial, as well as the liver, kidneys, lungs, spleen, and peripheral blood leukocytes (PBLs), were extracted from mice at indicated time-points post-injection for further experiments.

\section{Histopathology and Immunohistochemistry}

Cardiac tissues were fixed in formalin, and embedded in paraffin. Serially $4 \mu \mathrm{m}$-thick cardiac sections were stained with Hematoxylin and Eosin (H\&E) or Masson's trichrome (SigmaAldrich), and then were graded for severity of arteritis as previously. ${ }^{25}$ Immunohistochemical staining was performed with a purified goat anti-mouse VEGF-A polyclonal antibody (Ab) (R\&D System, Minneapolis, MN, USA), and monoclonal rabbit anti-mouse CD3 and Ly6G Abs (Abcam, Cambridge, MA, USA), and rat anti-mouse CD4, CD8, and CD11b Abs (BD Pharmingen, San Diego, CA, USA) for pan-T cell, neutrophil, helper-T, cytotoxic-T and monocyte/macrophage markers, respectively, as well as their corresponding isotypes. In brief, sections were deparaffinized, treated with $3 \%$ hydrogen peroxide to inactive endogenous peroxidase activity, and microwaved for $10 \mathrm{~min}$ in $10 \mathrm{mmol} / \mathrm{L}$ citrate buffers to retrieve antigens. The sections were then incubated with a primary $\mathrm{Ab}$ at $4^{\circ} \mathrm{C}$ overnight and detected with a secondary Ab using the SuperPicTure Polymer detection kit (Zymed Laboratories, South San Francisco, CA, USA) and 3,3-diaminobenzidine (DAB) chromogen (Invitrogen Life Technologies, Palo Alto, CA, USA).

\section{Western Blot Analysis}

Total proteins from murine organs/tissues were extracted by using the PRO-PREPTM (iNtRON Biotechnology, Seoul, Korea) protein extract solute, quantified by a Bradford-based assay (Bio-Rad Laboratories, Hercules, CA, USA), separated, and transferred, as previously. ${ }^{25}$ All blots were incubated overnight at $4{ }^{\circ} \mathrm{C}$ with each of the following Abs: a mouse monoclonal Ab for VEGF-A (Santa Cruz Biotechnology, Inc, San Diego, CA, USA) and for endothelial nitric oxide synthase (eNOS) (Transduction Laboratories, Lexington, KY, USA), the rat anti-mouse Abs for tumor necrosis factor (TNF)- $\alpha$ and IL-6 (BioLegend, Inc, San Diego, CA, USA), and a goat polyclonal $\mathrm{Ab}$ for dimethylarginine dimethylaminohydrolase (DDAH)-1 (Santa Cruz Biotechnology) and for DDAH-2 (Santa Cruz Biotechnology). Next, the blots were washed with tris-buffered saline with tween 20, incubated with horseradish peroxidase-coupled goat anti-mouse IgG for VEGF-A and eNOS, goat anti-rat IgG for TNF- $\alpha$ and IL-6, and donkey anti-goat IgG for DDAH-1 and DDAH-2, at room temperature for $1 \mathrm{~h}$, then developed using enhanced chemiluminescent detection (Amersham Pharmacia Biotech, Uppsala, Sweden), and finally exposed to film. Signals were quantified by densitometry (Quantity One Analysis software; Bio-Rad) as integrated optical density, and the results are shown as the ratio of VEGF-A, TNF- $\alpha$, IL-6, eNOS, DDAH-1, and DDAH-2 over glyceraldehyde-3-phosphate dehydrogenase (GAPDH). Both protein expression levels of GAPDH and $\beta$-actin were simultaneously validated in control- and treated-groups as being internal controls (Figure S1A).

\section{In Vitro Cell Culture}

RAW 264.7 cells (RAW cells), a murine monocyte/macrophage cell line (ATCC TIB-71; Bioresource Collection and Research Center, Taiwan), were maintained in complete RPMI 1640 medium (Gibco, Rockville, MD), as previously. ${ }^{25}$ Prior to the experiments, RAW cells at passage 7-10 were seeded in 6 -well plates $\left(2.5 \times 10^{5}\right.$ cells $\left./ \mathrm{ml}\right)$, and then treated either with LPS $(100 \mathrm{ng} / \mathrm{ml})$ or LCWE $(1 \mu \mathrm{g} / \mathrm{ml})$, and without stimulus for $6 \mathrm{~h}$ and $24 \mathrm{~h}$. For blocking studies, the RAW cells were pretreated for $30 \mathrm{~min}$ with BAY $(5 \mu \mathrm{mol} / \mathrm{L})$ before stimulation by LCWE. 
A
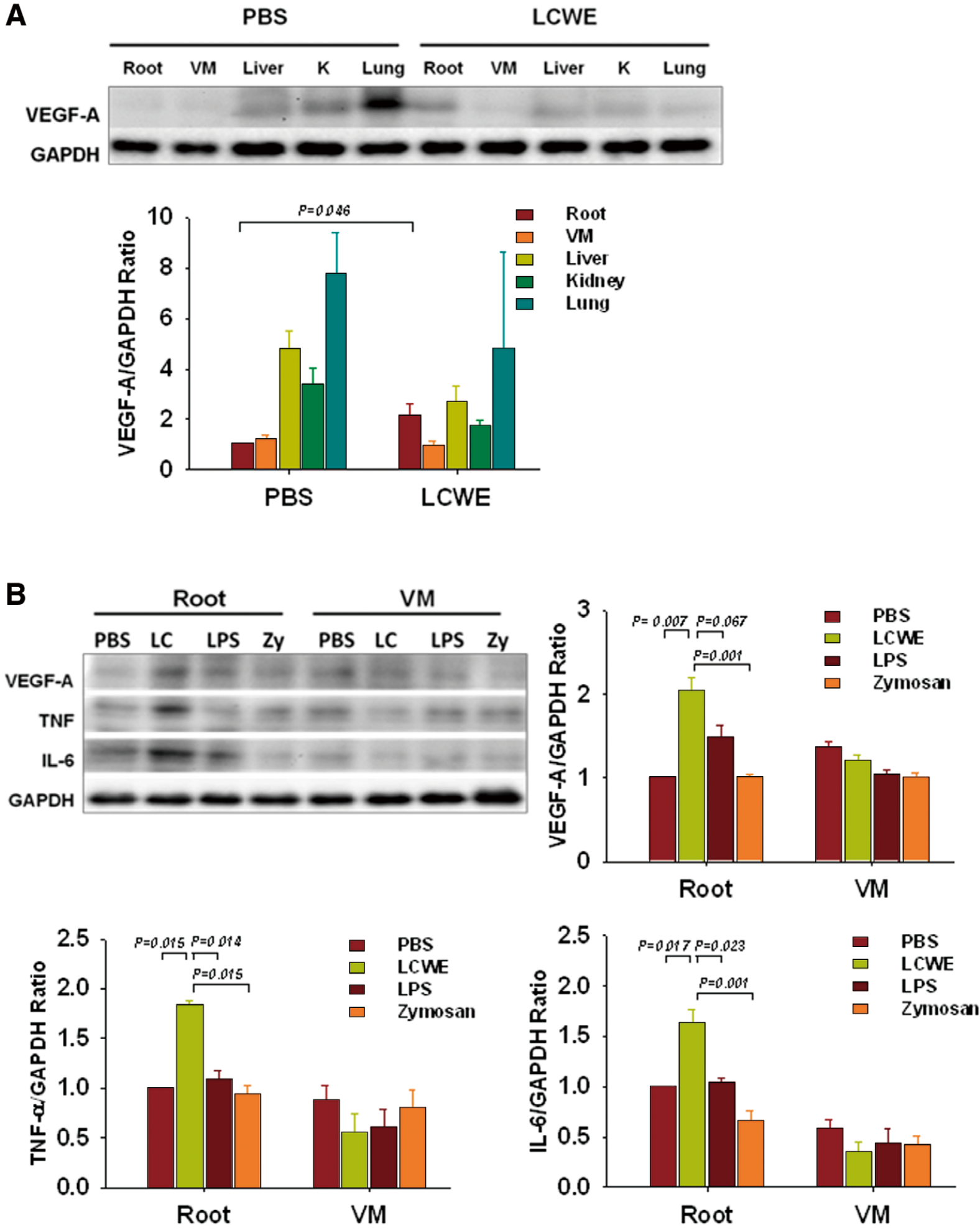

Figure 1. VEGF-A protein expression in murine tissues and organs 3 days after LCWE treatment. (A) Representative immunoblots of total proteins from the aortic roots (Root), ventricular myocardium (VM), liver, kidney (K) and lung showed VEGF-A protein levels in PBS- and LCWE-treated mice. (B) VEGF-A protein expression in aortic roots was significantly increased in LCWE (LC)-treated mice when compared to PBS-treated and zymosan (Zy)-treated mice. Furthermore, TNF- $a$ and IL-6 protein expression levels were significantly increased in LCWE-treated mice when compared to all other groups. No differential VEGF-A, TNF- $a$, or IL-6 expression was observed in the VM among these mice. VEGF, vascular endothelial growth factor; LCWE, Lactobacillus casei cell wall extract; PBS, phosphate buffered saline; TNF, tumor necrosis factor; IL-6, interleukin-6. 
A

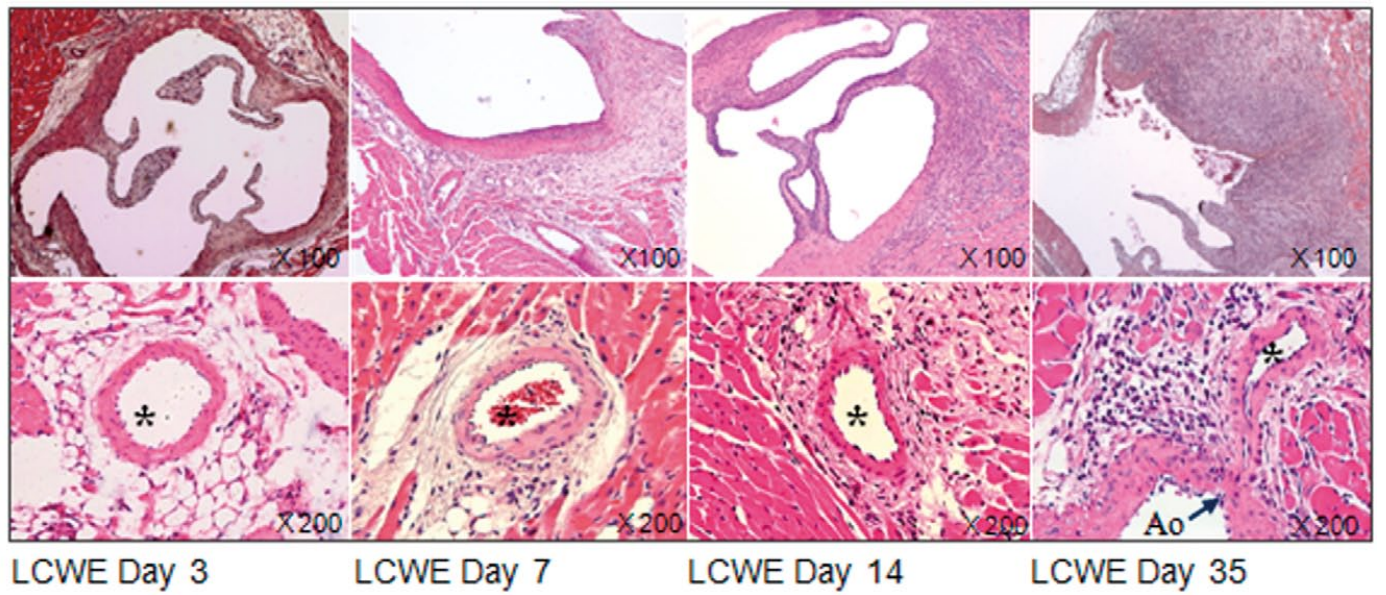

B

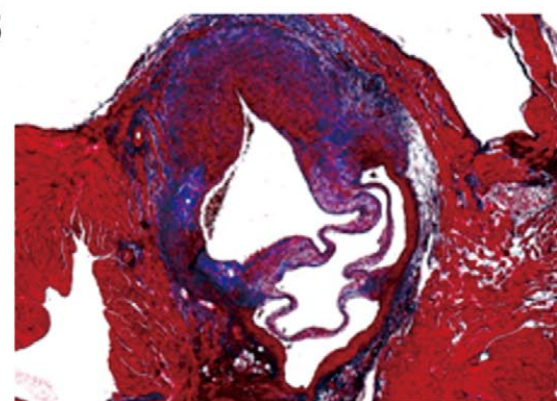

\section{D}

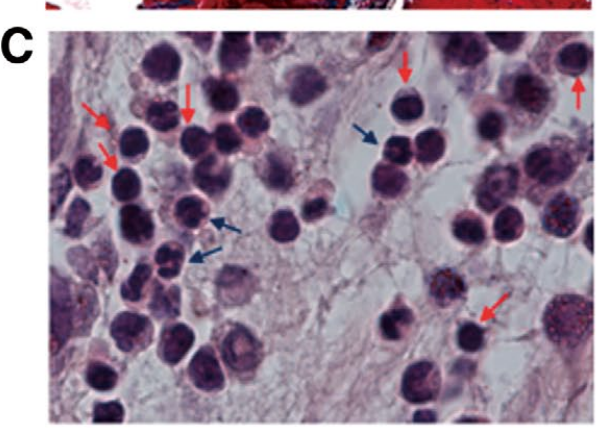

E


Figure 2. Cardiac histopathology in LCWE-treated mice. (A) Representative H\&E staining of aortic root/valve (Ao, upper panels) and coronary artery (CA, lower panels, marked by dark asterisks) from mice treated with LCWE at days 3, 7, 14, and 35 after injection, showing progressive infiltrates with subsequent vascular destruction with time. The blue arrow (right lower panel) indicated the narrowing of ostia of the CA (original magnification: $\times 100$ in upper panels; $\times 200$ in lower panels). (B) Trichrome staining indicated massive fibrosis surrounding the Ao 14 days after LCWE stimulation (original magnification: $\times 50$ ). (C) High magnification $(\times 1,000)$ by H\&E staining showed infiltrates of mononuclear cells (red arrows) and polymorphonuclear (segmented-nucleus) neutrophils (blue arrows). (D) A immunohistochemistry study showed the infiltrates of CD3-, CD4-, CD8-, CD11b- and Ly6Gpositive stained cells (original magnification: $\times 400$ in left panels, and $\times 200$ in right panels). (E) The quantitative data indicated the progressive severity of LCWE-induced CALs over time. LCWE, Lactobacillus casei cell wall extract; H\&E, Hematoxylin and Eosin; CALs, coronary artery lesions. 
A

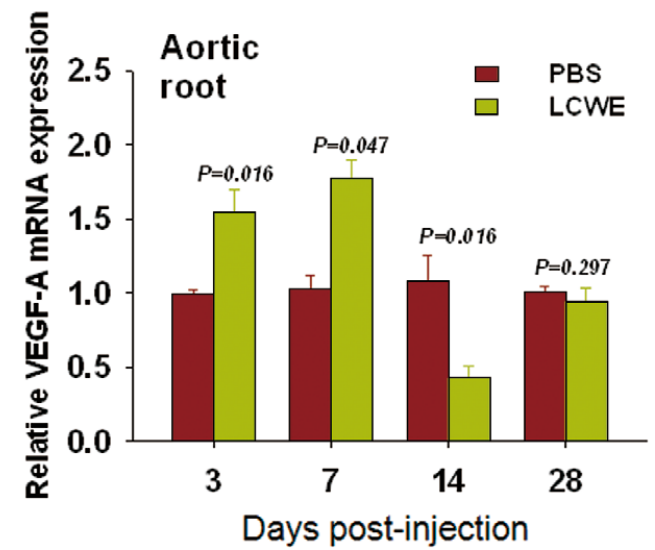

C

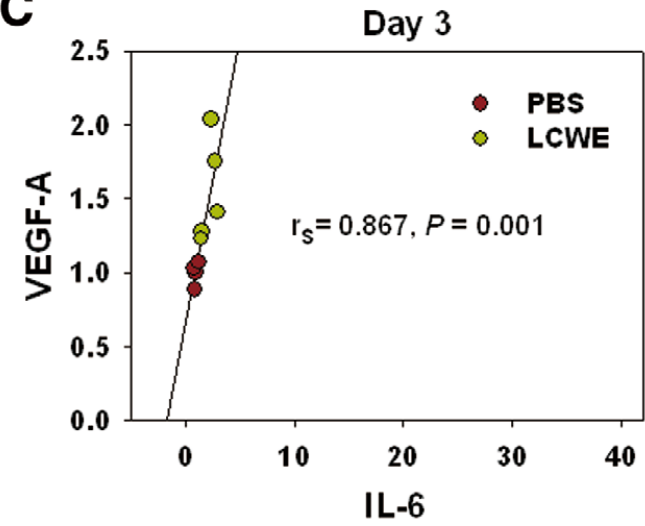

B

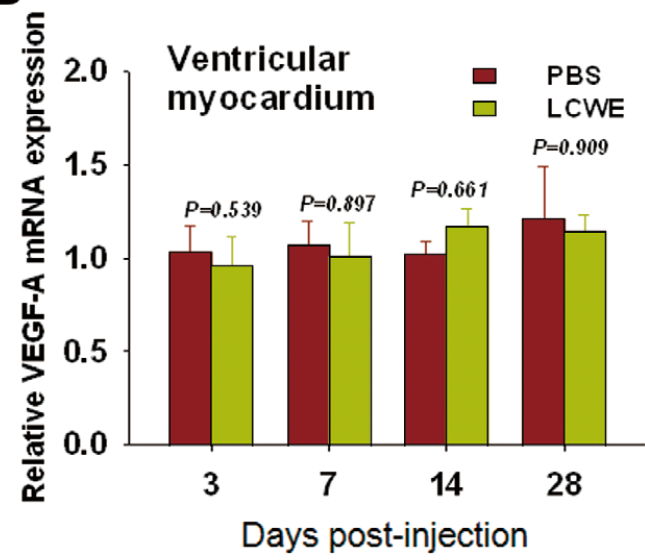

Day 14

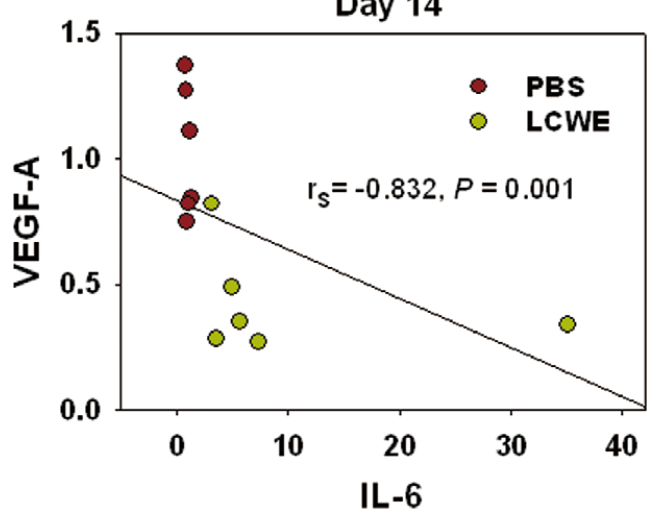

Figure 3. Dynamic alterations of VEGF-A mRNA levels in (A) aortic roots and (B) ventricular myocardium. (C) By Spearman correlation, VEGF-A and IL-6 mRNA levels from aortic roots were significantly positively correlated on day 3 but negatively correlated on day 14 in both PBS-treated and LCWE-treated mice. VEGF, vascular endothelial growth factor; IL-6, interleukin-6; PBS, phosphate buffered saline.

\section{RNA Isolation and Real-Time RT-Polymerase Chain Reaction (PCR)}

The aortic roots, VM and PBLs from mice, and RAW cells were homogenized in a TRIzol reagent (Invitrogen), and the RNA was obtained, as previously. ${ }^{25}$ Real-time PCR was then performed and optimized in the presence of SYBR green using an ABI 7500 Sequence Detection System (Applied Biosystems, Foster City, CA, USA). Sequences of primer pairs are shown in Table S1.

The amplified PCR products were expressed as a ratio in relation to the housekeeping-gene product, $\beta$-actin. Both mRNA levels of GAPDH and $\beta$-actin were simultaneously validated in control- and treated-groups as being internal controls (Figure S1B). The validation experiments were carried out in duplicate and amplification efficiencies were validated.

\section{Statistical Analysis}

Data are presented as mean \pm SEM. The relative amounts of mRNA, the severity of arteritis, and the ratios of VEGF-A, eNOS, DDAH-1, and DDAH-2 over GAPDH by Western blots, were analyzed by using the Mann-Whitney $U$ test between the 2 groups. Among the groups, a one-way ANOVA with Tukey's post-hoc test was used when equal variance was assumed, and a Games-Howell post-hoc test was used when equal variance was not assumed. A P value of $<0.05$ was considered statistically significant.

\section{Results}

\section{Early Increases in Aortic VEGF-A Expression After LCWE} Stimulation

To examine the spatial expression of VEGF-A in response to LCWE treatment, Western blot analysis was performed for all proteins in the aortic roots, ventricular myocardium (VM), liver, kidneys, and lungs, 3 days after injection $(n=3$ in each organ per group) (Figure 1). We found that, in PBS-treated mice, the liver, kidneys, and lungs had higher basal VEGF-A expression, whereas aortic roots and VM had a lower basal expression of VEGF-A (Figure 1A). In addition, compared to PBS-treated mice, LCWE-treated mice had significantly higher VEGF-A protein expression in the aortic roots. Apart from this, no differential expression of VEGF-A in the ventricular myocardia, liver, kidneys, or lungs was detected between LCWE- and PBS-treated mice. VEGF-A protein expression in 


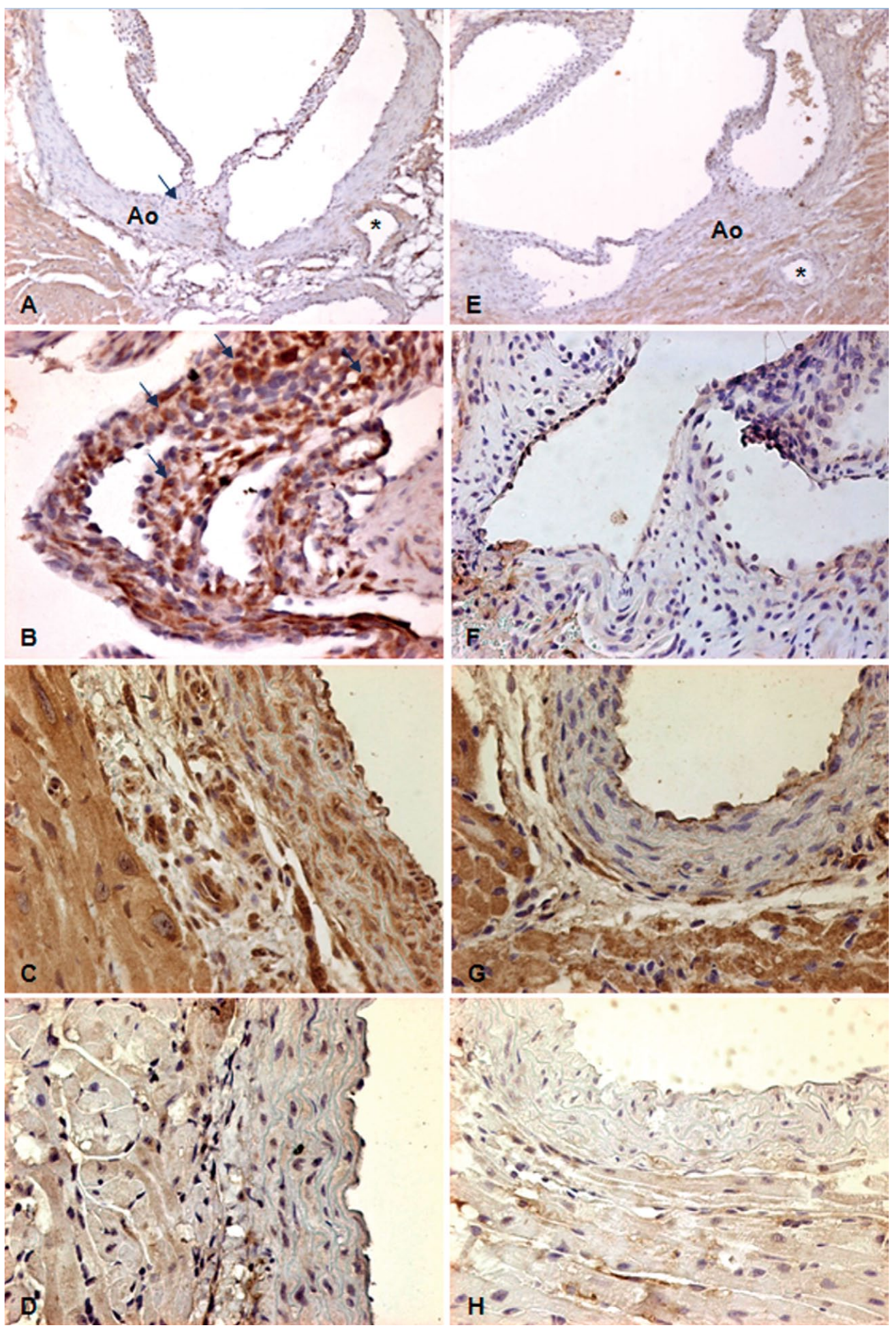

Figure 4. Immunohistochemical study of VEGF-A in the cardiac tissues 3 days after LCWE/or PBS treatment. (A-D) Cardiac slices from LCWE-treated mice reveal the presence of VEGF-A within the smooth muscle cells of the aortic ring and the neighboring ventricular myocardium, and (B) the infiltrating cells on the aortic valve (blue arrows). (E-H) Cardiac tissues from PBS-treated mice also revealed the presence of VEGF-A within the neighboring myocardium ( $E$ and $\mathbf{G}$ ) but less dominant over smooth muscle cells from the aortic ring $(\mathbf{G})$ when compared to those in LCWE-treated mice. An asterisk indicates the lumen of the coronary artery. $\mathbf{D}$ and $\mathbf{H}$ panels show staining of isotype control (original magnification: $\times 100$ in $\mathbf{A}$ and $\mathbf{E} ; \times 400$ in the other panels). VEGF, vascular endothelial growth factor; LCWE, Lactobacillus casei cell wall extract; PBS, phosphate buffered saline. 


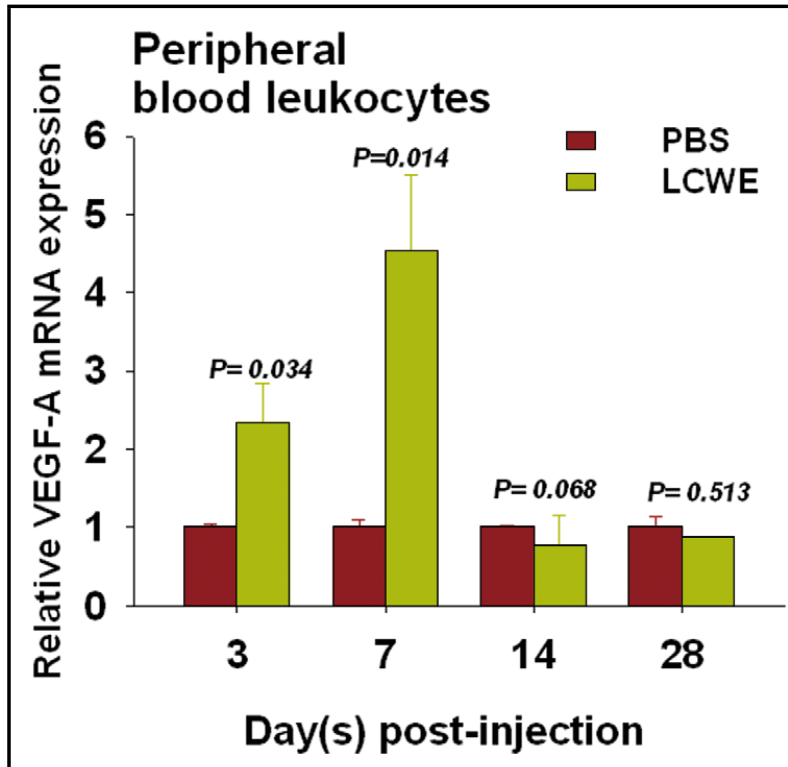

Figure 5. Increased VEGF-A mRNA expression in the peripheral blood leukocytes at days 3 and 7 after LCWE injection. VEGF, vascular endothelial growth factor; LCWE, Lactobacillus casei cell wall extract.

aortic roots was the highest in LCWE-treated mice when compared to that in PBS-, LPS-, and zymosan-treated mice (Figure 1B) ( $n=3$ in each group). Expression of TNF- $\alpha$ and IL-6 in aortic roots was significantly higher in LCWE-treated mice than in PBS-, LPS-, and zymosan-treated mice.

\section{Temporal Alteration of Histopathology and VEGF-A Expression}

All arteritis in LCWE-treated mice manifested as an increasing degree of infiltration of mononuclear cells and neutrophils into the perivascular/adventitial regions of the aorta, nearby proximal coronary arteries and ostia, as well as the neighboring myocardium between 3 and 35 days after injection $(n=5$ per time-point); this infiltration progressed to ostial occlusion with marked fibrinoid deposition at day 35 (Figures 2A-E), but little infiltrate was visible around distal coronary arteries. Fourteen days after treatment, some LCWE-treated mice exhibited proliferation of smooth muscle cells and thickness of the vascular wall of proximal coronary arteries, and some had tortuous and aneurysm-like vessels of proximal coronary arteries (Figure S2). We also examined the in vivo temporal effect of LCWE on VEGF-A production in the aortic roots and ventricular myocardia. The results showed that VEGF-A mRNA levels in aortic roots significantly increased on days 3 and 7 after LCWE injection, but significantly declined on day 14 after injection ( $\mathrm{n}=5-7$ for each group per time-point) (Figure 3A). VEGF-A expression was not differentially regulated in the VM between LCWE- and PBS-treated mice (Figure 3B).

Interestingly, analysis performed using Spearman's correlation found that the coefficient was 0.867 on day 3 ( $n=5$ each group), and -0.832 day 14 ( $n=6$ each group) after the injection showed significant correlations between IL- 6 and VEGF-A mRNA expression levels in the aortic roots of LCWE-treated mice and PBS-treated mice (Figure 3C).

\section{Microenvironment Distribution of VEGF-A in Aortic Roots}

To evaluate the local distribution of VEGF-A in LCWE-induced CALs, immunohistochemical staining of VEGF-A was

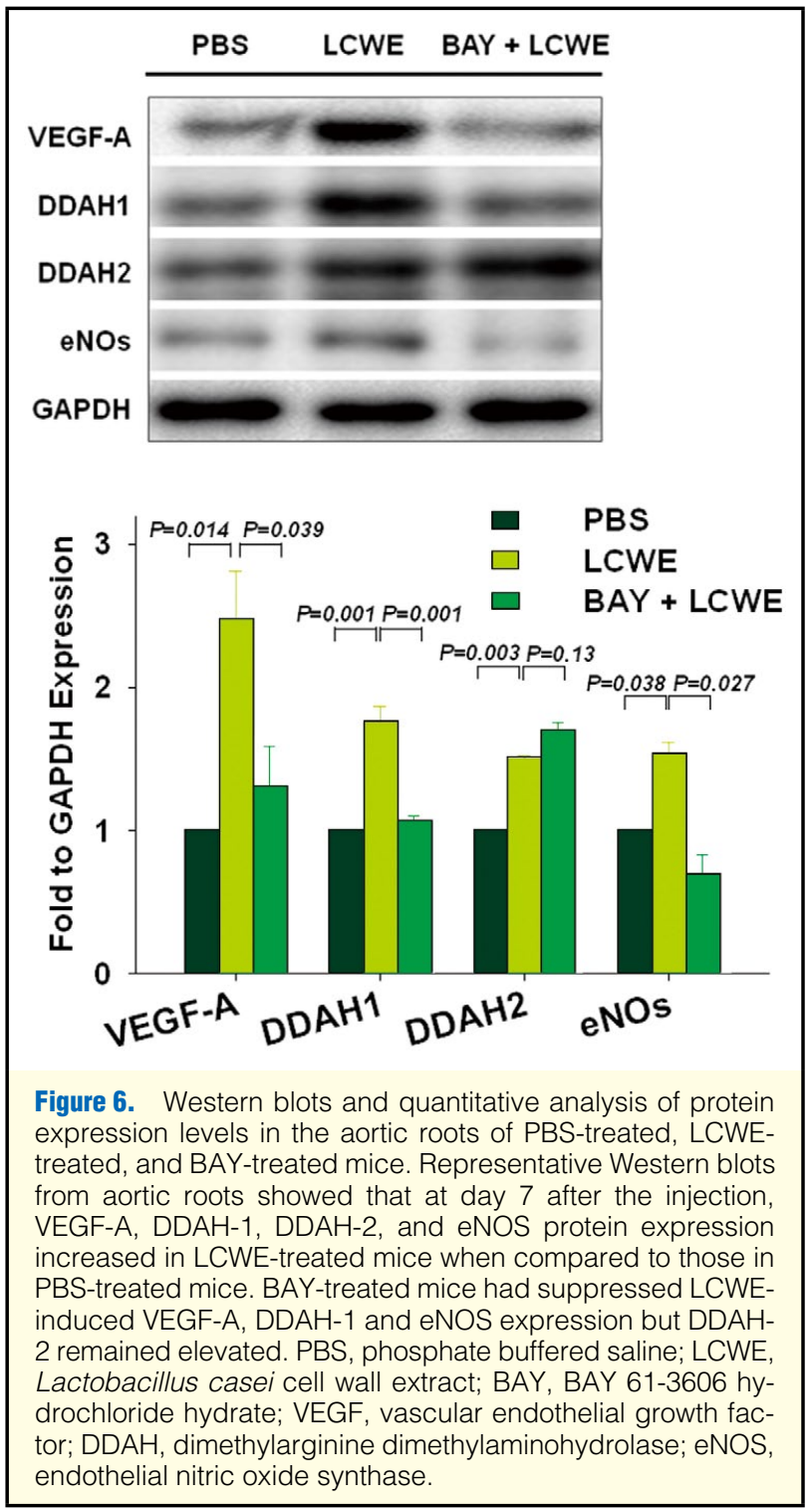

performed in the cardiac tissues 3 days after LCWE treatment, revealing the presence of VEGF-A expression in the smooth muscle cells of the aortic ring, the neighboring myocardium, VM, and the infiltrating cells on the aortic valve (Figure 4).

\section{Early Increase in VEGF-A Expression in PBLs}

The levels of VEGF-A mRNA in PBLs ( $n=5-7$ for each group per time-point) were significantly upregulated on days 3 and 7 after the LCWE injection, and showed no differences in expression on days 14 and 28 after the injection between LCWEtreated and PBS-treated mice (Figure 5).

\section{Attenuation of LCWE-Induced Aortic VEGF-A, DDAH-1, and eNOS Expression Accompanied by Augmentation of DDAH-2 Expression by Syk Inhibition}

To investigate whether VEGF-A signaling is involved in LCWE-induced CALs, in vivo VEGF-A expression in the aortic roots of PBS-treated, LCWE-treated, and BAY-treated mice were analyzed on day 7 after the injection ( $n=3$ in each group) (Figure 6). LCWE-induced VEGF-A expression in the aortic roots was significantly suppressed in the BAY-treated group. 

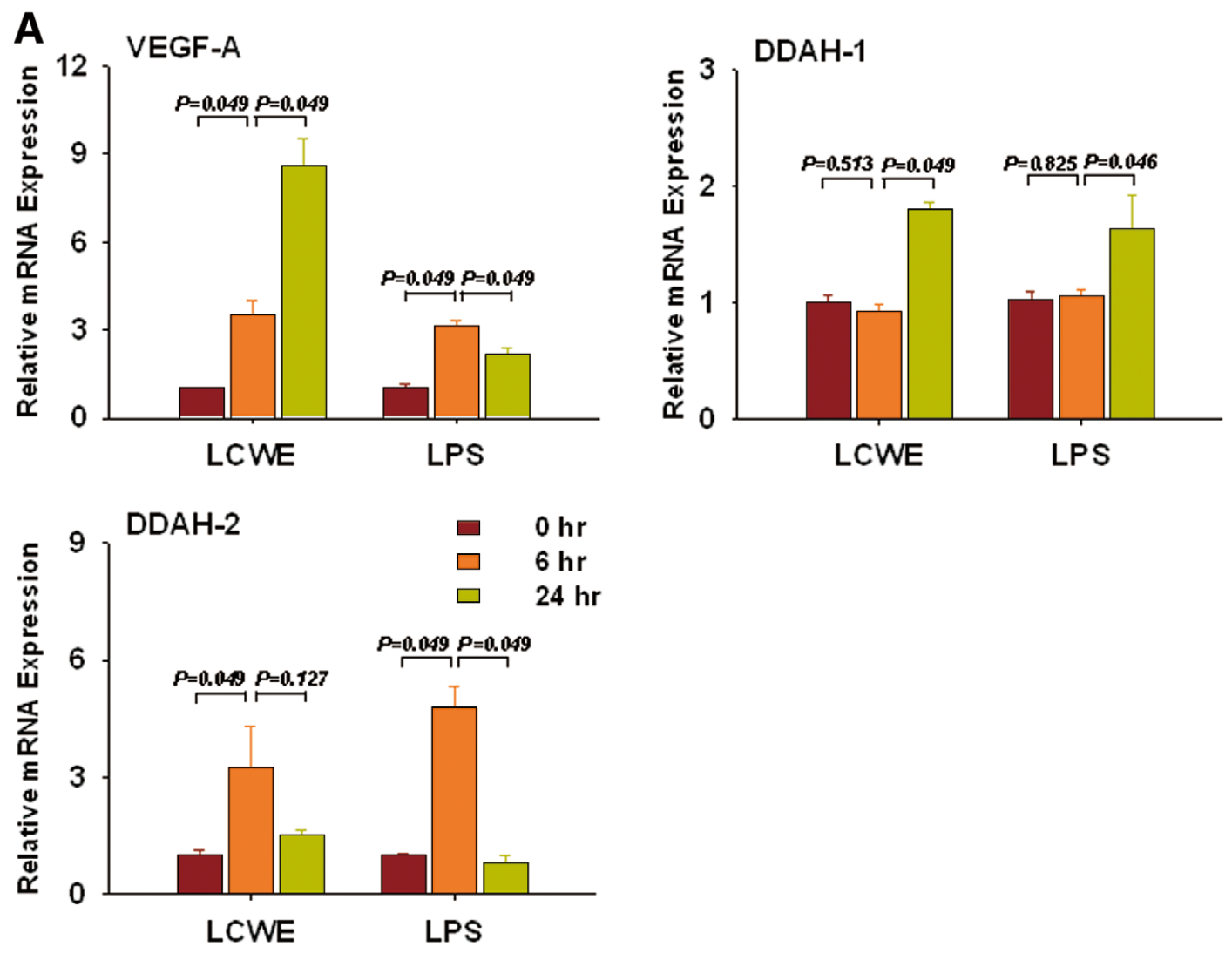

B

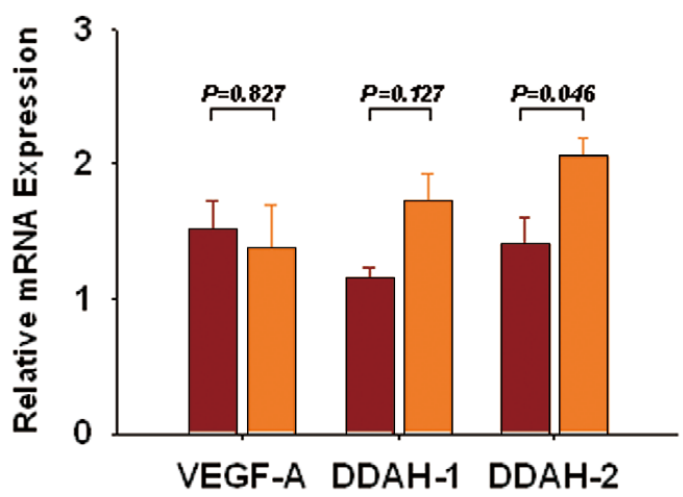

C

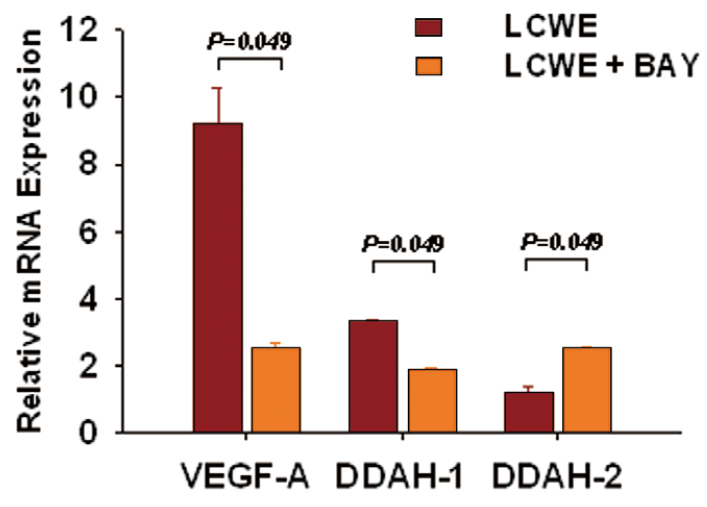

Figure 7. Relative mRNA levels of VEGF-A, DDAH-1, and DDAH-2 over $\beta$-actin by RAW cells. (A) Cells were stimulated for 0,6 , and $24 \mathrm{~h}$ either with LCWE or LPS. (B) Cells were pre-treated with or without BAY and then stimulated with LCWE for $6 \mathrm{~h}$ (left panel) and $24 \mathrm{~h}$ (right panel). VEGF, vascular endothelial growth factor; DDAH, dimethylarginine dimethylaminohydrolase; LCWE, Lactobacillus casei cell wall extract; LPS, lipopolysaccharide; BAY, BAY 61-3606 hydrochloride hydrate.

Aortic DDAH-1, DDAH-2, and eNOS expression significantly increased after LCWE treatment when compared to that in PBS-treated mice. Significant reductions in LCWE-induced DDAH-1 and eNOS expression were detected in the BAYtreated group compared to those in LCWE-treated group. However, aortic DDAH-2 remained elevated despite Syk inhibition $(\mathrm{P}=0.014$ comparing the PBS- and BAY-treated groups, using one-way ANOVA).
Reduction in LCWE-Induced Expression of VEGF-A and DDAH- 1 by RAW Cells Accompanied by Augmentation of DDAH-2 by Syk Inhibition

Macrophages have been shown to play a role in LCWE-induced CALs ${ }^{25}$ and atherosclerosis; ${ }^{33}$ therefore, we validated the effect of LCWE and Syk inhibition on VEGF-A regulation using RAW cells. In in vitro experiments, LCWE significantly upregulated VEGF-A, DDAH-1, and DDAH-2 mRNA production by RAW cells (Figure 7A). Such LCWE-induced 
VEGF-A and DDAH-1 upregulation was significantly suppressed by Syk inhibition (Figure 7C). In contrast, LCWEinduced DDAH-2 upregulation was significantly enhanced by Syk inhibition (Figures 7B,C).

\section{Discussion}

In this study, we demonstrated the spatial and temporal alteration of VEGF-A and its related signaling pathway in murine LCWE-induced CALs. KD-associated CALs are profoundly detrimental to human health. VEGF-A is an important chemokine in vascular disease and angiogenesis, and is reported to be strongly relevant to human $\mathrm{KD}{ }^{14-19,21,34}$ therefore, it is necessary to elucidate the local involvement of VEGF-A in KD-associated CALs. Because of the difficulty in obtaining cardiac samples from human patients with $\mathrm{KD}$, we attempted to explore this relationship using a murine model of LCWEinduced CALs, which mimic CALs in human KD. ${ }^{29}$

In in vivo experiments, LCWE induced early VEGF-A expression in aortic roots and PBLs but not in the VM, liver, kidneys, or lungs. Importantly, this early aortic increase in VEGF-A expression was suppressed in the BAY-treated "arteritis-ameliorated" group (Figures 5,S3). VEGF-A involvement differs between tissues and organs during the various stages of human KD, as well as during the various stages of LCWE-induced CALs. During the early acute phase of KD, production of VEGF-A in peripheral blood mononuclear cells markedly increases, especially in patients with CALs. ${ }^{19}$ In autopsied patients with a history of KD, VEGF-A expression has been observed within smooth muscle cells; VEGF-A is particularly highly expressed in the newly formed microvessels within the intima of affected human hearts in the later chronic inflammation stages of the disease. ${ }^{5}$ In our study, similar to these findings in human KD, VEGF-A production by PBLs in LCWEtreated mice was significantly augmented on days 3 and 7 after injection. In our immunohistochemical analysis, only a few VEGF-stained infiltrates were detected in the aortic valve during the early phase after LCWE stimulation. The early increase in aortic VEGF-A was attributed to VEGF-A production by the smooth muscle cells of the aortic ring and coronary arteries, and even by the neighboring myocardium. However, this is difficult to validate in human patients with acute KD.

The relationship between VEGF-A and LCWE-induced CALs and human KD remains an important area of study. Gene expression of VEGF-A is regulated by various stimuli such as hypoxia, interleukin (IL)- $1, \mathrm{TNF}-\alpha$, transforming growth factor- $\beta 1$, nitric oxide, DDAH- 1 , and DDAH- $2 .{ }^{35-38}$ We previously reported that systemic plasma levels of TNF- $\alpha$, IL-6, and MCP-1 rapidly increased soon after LCWE stimulation, ${ }^{30}$ and that Syk inhibition significantly suppressed plasma levels of IL-6 and MCP-1, reducing the severity of LCWE-induced $\mathrm{CALs}^{25}$ and TNF- $\alpha$ and VEGF-A expression in aortic roots (Figure S4). TNF- $\alpha$ plays a crucial role in mediating the development of LCWE-induced CALs. ${ }^{39}$ Hojo et al. ${ }^{40}$ reported that IL-6 plays a role in regulating local VEGF-A production by interaction between human monocytes and smooth muscle cells. Therefore, it appears reasonable that such LCWE-induced systemic robust proinflammatory immune responses and/or local TNF- $\alpha$ and IL-6 are responsible for local VEGFA upregulation in the aortic roots. Similar to the report by Okitsu-Negishi et al, ${ }^{32}$ we found that LPS was unable to induce CALs in mice. LPS-treated mice tended to develop higher plasma levels of TNF- $\alpha$, IL-6, and MCP-1 and slightly increased IL-1 $\beta$ levels (Figure S5) soon after LCWE treatment, but had lower levels of local aortic TNF- $\alpha$, IL-6, and VEGF-A
(Figure 1B) compared to those in LCWE-treated mice. Such spatial discrepancies between local aortic roots and systemic circulation in the expression of TNF- $\alpha$ and IL- 6 emphasized the importance of local regulation within the microenvironment of the aortic roots, as well as other possible contributory factors. Additionally, we demonstrated that LCWE-treated mice had continuing increases in aortic IL-6 mRNA expression from days 3 to 14, reflecting pathologic disease progression from no obvious infiltrates to massive inflammatory infiltrates; furthermore, their aortic TNF- $\alpha$ mRNA reached maximum levels on the seventh day after LCWE injection. ${ }^{25}$ Aortic VEGF-A mRNA levels showed an early positive and a late negative relationship with the IL-6 mRNA level; this finding might be attributable to the differential involvement of VEGF-A in different stage of CALs. IL-6 is a pro-fibrotic regulatory factor, mediating cardiac fibrosis in response to angiotensin II infusion. ${ }^{41}$ Therefore, we speculate that an early increase in VEGF-A expression occurs during the process of acute inflammation, and that the later decrease in VEGF-A expression and the associated imbalance between pro-angiogenic VEGF-A and pro-fibrotic IL-6 contribute to subsequent fibrotic changes in CALs. Taken together, these findings suggest that, in addition to systemic and local aortic TNF- $\alpha$ and IL-6, other factors also play a crucial role in mediating local VEGF-A regulation in LCWE-induced CALs.

Both DDAH-1 and DDAH-2, which are both responsible for the metabolism of asymmetric dimethylarginine, ${ }^{42}$ can be used to determine bioavailable nitric oxide, ${ }^{43}$ and are reported to be involved in some conditions related to increased cardiovascular risk, vascular repair, ${ }^{44}$ and VEGF-A regulation. ${ }^{35,37,45}$ DDAH-1 is predominantly expressed in the liver, kidneys and aorta, whereas DDAH-2 is predominantly distributed in the endothelium, at the site of eNOS expression, blood vessels, heart, and immune tissues..$^{35}$ eNOS, an important down-stream mediator of VEGF-A, performs multiple functions in the blood vessels, including endothelial cell proliferation, recruitment and functioning of endothelial progenitor cells, and endothelium-dependent vasorelaxation. ${ }^{46}$ Our in vitro data showed that LCWE significantly upregulates RAW cells to produce VEGF-A, DDAH-1, and DDAH-2, as does LPS. Furthermore, LCWEinduced VEGF-A and DDAH-2 were significantly higher than those stimulated by LPS $24 \mathrm{~h}$ after treatment (both $\mathrm{P}=0.049$ ). When compared to the mRNA levels $6 \mathrm{~h}$ and $24 \mathrm{~h}$ after treatment, Syk inhibition appeared to directly upregulate DDAH-2 but indirectly downregulate VEGF-A and DDAH-1. Based on our in vivo results, it appears reasonable to suggest that LCWE-induced aortic expression of DDAH-2 via an unknown mechanism upregulates VEGF-A expression, which may then stimulate further vascular repair. Although asymmetric dimethylarginine might be involved in this LCWE-induced DDAH-1 and DDAH-2 expression, we did not examine asymmetric dimethylarginine levels, DDAH enzyme activity, or oxidative stress status because of the limited amount of protein we were able to obtain from each aortic root. In contrast to this, in the BAY-treated arteritis-ameliorated group, the suppressed elevation of VEGF-A and eNOS may reflect the reduced need for vascular repair because of the reduced severity of CALs. Aortic DDAH-1 expression decreased in line with VEGF-A and eNOS suppression, but DDAH-2 expression was even more enhanced in the BAY-treated group. Although the role of Syk in angiotensin II-induced VEGF-mediated vasculogenesis has been established, ${ }^{47}$ there is still inadequate evidence to speculate about the effect of Syk on DDAH-1 and DDAH-2 in the pathogenesis of LCWE-induced CALs. DDAH-2 might exert some additional effects to overcome LCWE-induced in- 
flammation, which negatively regulates VEGF-A in an indirect manner.

This study has several limitations. First, we did not assess the determinant role of VEGF-A, DDAH-1, DDAH-2, and Syk in LCWE-induced CALs in vivo. Although our results indicate the involvement of aortic VEGF-A signaling and regulation in LCWE-induced CALs, further studies should investigate whether aortic VEGF-A plays a determinant role or is merely a concomitant phenomenon in LCWE-induced CALs. It would be beneficial to verify the role of VEGF-A in the pathogenesis of LCWE-induced CALs, possibly by using VEGF-A or VEGFR knockout mice, or even tissue-specific VEGF-A-deficient mice. In addition, because $\mathrm{KD}$ is a systemic vasculitis disease, showing the worst lesions on coronary arteries, future research should examine the expression of VEGF-A in other blood vessels, such as the renal arteries, mesenteric arteries and iliac arteries, in more detail. Further analysis should address the differential expression of VEGF-A in different arterial blood vessels. Finally, we did not investigate VEGF-A regulation and signaling by using other component cells of aortic roots, such as endothelial cells and smooth muscle cells, because expressions of DDAH-1 and DDAH-2 are cell-type specific.

\section{Conclusions}

CALs in KD are a serious problem because of persistent vascular inflammation and remodeling with an unclear pathogenesis. Increased aortic VEGF-A expression and the related DDAH-1, DDAH-2, and eNOS elevations were associated with LCWE-induced murine CALs. In light of the strong association between VEGF and human $\mathrm{KD}$, it is imperative to investigate VEGF-A regulation and signaling pathways in murine CALs and to use these study findings to develop a more effective therapeutic approach for improving cardiovascular outcomes in KD patients with CALs.

\section{Acknowledgments}

This study was, in part, supported by grants CMRPG881041 and CMRPG881042 from Kaohsiung Chang Gung Memorial Hospital, and grants CCF100-03 and CCFT0805 from Cardiac Children's Foundation Taiwan for Dr Lin, and by grants NSC 97-2314-B-182A-054-MY2 and NSC 100-2314-B-182A-048-MY3 from the National Science Council, and NHRI-100AI-PDCO-0909111 from the National Health Research Institutes, Taiwan for Dr Yang.

\section{Disclosures}

None.

\section{References}

1. Kawasaki T, Kosaki F, Okawa S, Shigematsu I, Yanagawa H. A new infantile acute febrile mucocutaneous lymph node syndrome (MLNS) prevailing in Japan. Pediatrics 1974; 54: 271-276.

2. Barron KS. Kawasaki disease in children. Curr Opin Rheumatol 1998; 10: $29-37$

3. Iemura M, Ishii M, Sugimura T, Akagi T, Kato H. Long term consequences of regressed coronary aneurysms after Kawasaki disease: Vascular wall morphology and function. Heart 2000; 83: 307-311.

4. Kato H, Sugimura T, Akagi T, Sato N, Hashino K, Maeno Y, et al. Long-term consequences of Kawasaki disease. A 10- to 21-year followup study of 594 patients. Circulation 1996; 94: 1379-1385.

5. Suzuki A, Miyagawa-Tomita S, Komatsu K, Nishikawa T, Sakomura Y, Horie T, et al. Active remodeling of the coronary arterial lesions in the late phase of Kawasaki disease: Immunohistochemical study. Circulation 2000; 101: 2935-2941.

6. Tsuda E, Arakaki Y, Shimizu T, Sakaguchi H, Yoshimura S, Yazaki $\mathrm{S}$, et al. Changes in causes of sudden deaths by decade in patients with coronary arterial lesions due to Kawasaki disease. Cardiol Young 2005; 15: 481-488.

7. Takahashi K, Oharaseki T, Yokouchi Y, Yamada H, Shibuya K,
Naoe S. A half-century of autopsy results: Incidence of pediatric vasculitis syndromes, especially Kawasaki disease. Circ J 2012; 76: 964-970.

8. Nagy JA, Dvorak AM, Dvorak HF. VEGF-A(164/165) and PIGF: Roles in angiogenesis and arteriogenesis. Trends Cardiovasc Med 2003; 13: $169-175$.

9. Takahashi H, Shibuya M. The vascular endothelial growth factor (VEGF)/VEGF receptor system and its role under physiological and pathological conditions. Clin Sci (Lond) 2005; 109: 227-241.

10. Melder RJ, Koenig GC, Witwer BP, Safabakhsh N, Munn LL, Jain RK. During angiogenesis, vascular endothelial growth factor and basic fibroblast growth factor regulate natural killer cell adhesion to tumor endothelium. Nat Med 1996; 2: 992-997.

11. Kim I, Moon SO, Kim SH, Kim HJ, Koh YS, Koh GY. Vascular endothelial growth factor expression of intercellular adhesion molecule 1 (ICAM-1), vascular cell adhesion molecule 1 (VCAM-1), and E-selectin through nuclear factor-kappa $\mathrm{B}$ activation in endothelial cells. J Biol Chem 2001; 276: 7614-7620.

12. Barleon B, Sozzani S, Zhou D, Weich HA, Mantovani A, Marme D. Migration of human monocytes in response to vascular endothelial growth factor (VEGF) is mediated via the VEGF receptor flt-1. Blood 1996; 87: 3336-3343.

13. Clauss M. Functions of the VEGF receptor-1 (FLT-1) in the vasculature. Trends Cardiovasc Med 1998; 8: 241-245.

14. Kariyazono H, Ohno T, Khajoee V, Ihara K, Kusuhara K, Kinukawa $\mathrm{N}$, et al. Association of vascular endothelial growth factor (VEGF) and VEGF receptor gene polymorphisms with coronary artery lesions of Kawasaki disease. Pediatr Res 2004; 56: 953-959.

15. Yasukawa K, Terai M, Shulman ST, Toyozaki T, Yajima S, Kohno Y, et al. Systemic production of vascular endothelial growth factor and fms-like tyrosine kinase-1 receptor in acute Kawasaki disease. Circulation 2002; 105: 766-769.

16. Terai M, Yasukawa K, Narumoto S, Tateno S, Oana S, Kohno Y. Vascular endothelial growth factor in acute Kawasaki disease. Am J Cardiol 1999; 83: 337-339.

17. Ohno T, Igarashi H, Inoue K, Akazawa K, Joho K, Hara T. Serum vascular endothelial growth factor: A new predictive indicator for the occurrence of coronary artery lesions in Kawasaki disease. Eur $J$ Pediatr 2000; 159: 424-429.

18. Maeno N, Takei S, Masuda K, Akaike H, Matsuo K, Kitajima I, et al. Increased serum levels of vascular endothelial growth factor in Kawasaki disease. Pediatr Res 1998; 44: 596-599.

19. Hamamichi Y, Ichida F, Yu X, Hirono KI, Uese KI, Hashimoto I, et al. Neutrophils and mononuclear cells express vascular endothelial growth factor in acute Kawasaki disease: Its possible role in progression of coronary artery lesions. Pediatr Res 2001; 49: 74-80.

20. Terai M, Jibiki T, Harada A, Terashima Y, Yasukawa K, Tateno S, et al. Dramatic decrease of circulating levels of monocyte chemoattractant protein-1 in Kawasaki disease after gamma globulin treatment. J Leukoc Biol 1999; 65: 566-572.

21. Breunis WB, Davila S, Shimizu C, Oharaseki T, Takahashi K, van Houdt M, et al. Disruption of vascular homeostasis in patients with Kawasaki disease: Involvement of vascular endothelial growth factor and angiopoietins. Arthritis Rheum 2012; 64: 306-315.

22. Schafer G, Cramer T, Suske G, Kemmner W, Wiedenmann B, Hocker M. Oxidative stress regulates vascular endothelial growth factor-A gene transcription through Sp1- and Sp3-dependent activation of two proximal GC-rich promoter elements. J Biol Chem 2003; 278: 81908198.

23. Yahata T, Suzuki C, Hamaoka A, Fujii M, Hamaoka K. Dynamics of reactive oxygen metabolites and biological antioxidant potential in the acute stage of Kawasaki disease. Circ J 2011; 75: 2453-2459.

24. Takatsuki S, Ito Y, Takeuchi D, Hoshida H, Nakayama T, Matsuura $\mathrm{H}$, et al. IVIG reduced vascular oxidative stress in patients with Kawasaki disease. Circ J 2009; 73: 1315-1318.

25. Lin IC, Suen JL, Huang SK, Huang SC, Huang HC, Kuo HC, et al. Dectin-1/Syk signaling is involved in Lactobacillus casei cell wall extract-induced mouse model of Kawasaki disease. Immunobiology 2013; 218: 201-212.

26. Wang X, Mychajlowycz M, Lau C, Gutierrez C, Scott JA, Chow CW. Spleen tyrosine kinase mediates BEAS-2B cell migration and proliferation and human rhinovirus-induced expression of vascular endothelial growth factor and interleukin-8. J Pharmacol Exp Ther 2012; 340: $277-285$.

27. Kazerounian S, Duquette M, Reyes MA, Lawler JT, Song K, Perruzzi $\mathrm{C}$, et al. Priming of the vascular endothelial growth factor signaling pathway by thrombospondin-1, CD36, and spleen tyrosine kinase. Blood 2011; 117: 4658-4666.

28. Simon DI. Inflammation and vascular injury: Basic discovery to drug development. Circ J 2012; 76: 1811-1818. 
29. Lehman TJ, Walker SM, Mahnovski V, McCurdy D. Coronary arteritis in mice following the systemic injection of group B Lactobacillus casei cell walls in aqueous suspension. Arthritis Rheum 1985; 28: $652-659$.

30. Lin IC, Kuo HC, Lin YJ, Wang FS, Wang L, Huang SC, et al. Augmented TLR2 expression on monocytes in both human Kawasaki Disease and a mouse model of coronary arteritis. PLoS One 2012; 7: e38635, doi:10.1371/journal.pone.0038635 (accessed on June 21, 2012).

31. Dubois M, Gilles K, Hamilton JK, Rebers PA, Smith F. Colorimetric method for the determination of sugars and related substances. Anal Chem 1956; 28: 350-356.

32. Okitsu-Negishi S, Nakano I, Suzuki K, Hashira S, Abe T, Yoshino $\mathrm{K}$. The induction of cardioangitis by Lactobacillus casei cell wall in mice. I. The cytokine production from murine macrophages by Lactobacillus casei cell wall extract. Clin Immunol Immunopathol 1996; 78: $30-40$.

33. Fenyo IM, Gafencu AV. The involvement of the monocytes/macrophages in chronic inflammation associated with atherosclerosis. Immunobiology 2013; 218: 1376-1384.

34. Ohno T, Yuge T, Kariyazono H, Igarashi H, Joh-o K, Kinugawa N, et al. Serum hepatocyte growth factor combined with vascular endothelial growth factor as a predictive indicator for the occurrence of coronary artery lesions in Kawasaki disease. Eur J Pediatr 2002; 161: $105-111$.

35. Palm F, Onozato ML, Luo Z, Wilcox CS. Dimethylarginine dimethylaminohydrolase (DDAH): Expression, regulation, and function in the cardiovascular and renal systems. Am J Physiol Heart Circ Physiol 2007; 293: H3227-H3245.

36. Hasegawa K, Wakino S, Tanaka T, Kimoto M, Tatematsu S, Kanda $\mathrm{T}$, et al. Dimethylarginine dimethylaminohydrolase 2 increases vascular endothelial growth factor expression through Sp1 transcription factor in endothelial cells. Arterioscler Thromb Vasc Biol 2006; 26: $1488-1494$.

37. Fiedler LR, Bachetti T, Leiper J, Zachary I, Chen L, Renne T, et al. The ADMA/DDAH pathway regulates VEGF-mediated angiogenesis. Arterioscler Thromb Vasc Biol 2009; 29: 2117-2124.

38. Kostourou V, Robinson SP, Cartwright JE, Whitley GS. Dimethylarginine dimethylaminohydrolase I enhances tumour growth and angiogenesis. Br J Cancer 2002; 87: 673-680.

39. Hui-Yuen JS, Duong TT, Yeung RS. TNF-alpha is necessary for induction of coronary artery inflammation and aneurysm formation in an animal model of Kawasaki disease. J Immunol 2006; 176: 6294-6301.

40. Hojo Y, Ikeda U, Maeda Y, Takahashi M, Takizawa T, Okada M, et al. Interaction between human monocytes and vascular smooth muscle cells induces vascular endothelial growth factor expression. Atherosclerosis 2000; 150: $63-70$.

41. Ma F, Li Y, Jia L, Han Y, Cheng J, Li H, et al. Macrophage-stimulated cardiac fibroblast production of IL-6 is essential for TGF beta/ Smad activation and cardiac fibrosis induced by angiotensin II. PLoS One 2012; 7: e35144.
42. Achan V, Broadhead M, Malaki M, Whitley G, Leiper J, MacAllister $\mathrm{R}$, et al. Asymmetric dimethylarginine causes hypertension and cardiac dysfunction in humans and is actively metabolized by dimethylarginine dimethylaminohydrolase. Arterioscler Thromb Vasc Biol 2003; 23: $1455-1459$.

43. Dayoub H, Achan V, Adimoolam S, Jacobi J, Stuehlinger MC, Wang BY, et al. Dimethylarginine dimethylaminohydrolase regulates nitric oxide synthesis: Genetic and physiological evidence. Circulation 2003; 108: $3042-3047$.

44. Konishi H, Sydow K, Cooke JP. Dimethylarginine dimethylaminohydrolase promotes endothelial repair after vascular injury. J Am Coll Cardiol 2007; 49: 1099-1105.

45. Smith CL, Birdsey GM, Anthony S, Arrigoni FI, Leiper JM, Vallance P. Dimethylarginine dimethylaminohydrolase activity modulates ADMA levels, VEGF expression, and cell phenotype. Biochem Biophys Res Commun 2003; 308: 984-989.

46. Kroll J, Waltenberger J. VEGF-A induces expression of eNOS and iNOS in endothelial cells via VEGF receptor-2 (KDR). Biochem Biophys Res Commun 1998; 252: 743-746.

47. Buharalioglu CK, Song CY, Yaghini FA, Ghafoor HU, Motiwala M, Adris T, et al. Angiotensin II-induced process of angiogenesis is mediated by spleen tyrosine kinase via VEGF receptor-1 phosphorylation. Am J Physiol Heart Circ Physiol 2011; 301: H1043-H1055.

\section{Supplementary Files}

Supplementary File 1

Figure S1. (A) Protein and (B) mRNA expression of GAPDH and $\beta$-actin as internal controls.

Figure S2. (A) Some LCWE-treated mice exhibited proliferation of smooth muscle cells and thickness of the vascular wall of proximal coronary arteries (CA) near the ostium, 14 days after LCWE treatment.

Figure S3. (A) Cardiac histopathology of H\&E staining in LCWEtreated and BAY-treated mice 7 days after LCWE treatment.

Figure S4. Protein expression levels of TNF- $\alpha$ in the aortic roots of mice 7 days after treatment with PBS, LCWE, and BAY+LCWE ( $\mathrm{n}=$ 3 each group, ${ }^{*} \mathrm{P}<0.05$ by one-way ANOVA post-hoc modification).

Figure S5. Plasma levels of tumor necrosis factor (TNF)- $\alpha$, interleukin (IL)-6, monocyte chemoattractant protein (MCP)-1, and IL-1 $\beta$ in mice, 3 days after treatment with phosphate buffered saline (PBS), Lactobacillus casei cell wall extract (LCWE), and lipopolysaccharide (LPS) ( $\mathrm{n}=6$ each group, compared by one-way ANOVA posthoc modification).

Table S1. Sequences of primer pairs for each target gene

Please find supplementary file(s);

http://dx.doi.org/10.1253/circj.CJ-13-0612 